\title{
A Framework for Lattice QCD Calculations on GPUs
}

\author{
F. T. Winter*, M. A. Clark ${ }^{\dagger}$, R. G. Edwards*, B. Joó* \\ *Thomas Jefferson National Accelerator Facility, Newport News, VA, USA \\ \{fwinter,edwards,bjoo\}@jlab.org \\ ${ }^{\dagger}$ NVIDIA Corporation, 2701 San Tomas Expressway, Santa Clara, CA 95050, USA \\ mclark@nvidia.com
}

\begin{abstract}
Computing platforms equipped with accelerators like GPUs have proven to provide great computational power. However, exploiting such platforms for existing scientific applications is not a trivial task. Current GPU programming frameworks such as CUDA $\mathrm{C} / \mathrm{C}++$ require low-level programming from the developer in order to achieve high performance code. As a result porting of applications to GPUs is typically limited to timedominant algorithms and routines, leaving the remainder not accelerated which can open a serious Amdahl's law issue.

The lattice QCD application Chroma allows to explore a different porting strategy. The layered structure of the software architecture logically separates the data-parallel from the application layer. The QCD Data-Parallel software layer provides data types and expressions with stencil-like operations suitable for lattice field theory and Chroma implements algorithms in terms of this high-level interface. Thus by porting the low-level layer one can effectively move the whole application in one swing to a different platform.

The QDP-JIT/PTX library, the reimplementation of the lowlevel layer, provides a framework for lattice QCD calculations for the CUDA architecture. The complete software interface is supported and thus applications can be run unaltered on GPUbased parallel computers. This reimplementation was possible due to the availability of a JIT compiler (part of the NVIDIA Linux kernel driver) which translates an assembly-like language (PTX) to GPU code. The expression template technique is used to build PTX code generators and a software cache manages the GPU memory.

This reimplementation allows us to deploy an efficient implementation of the full gauge-generation program with dynamical fermions on large-scale GPU-based machines such as Titan and Blue Waters which accelerates the algorithm by more than an order of magnitude.
\end{abstract}

\section{INTRODUCTION}

Recent trends in HPC computer architecture are making massively multicore parallelism and heterogeneity omnipresent. Computer centers around the globe are deploying supercomputers utilizing hybrid architectures accelerated with Graphic Processing Units (GPUs).

NVIDIA established CUDA as a parallel computing platform and programming model which enables developers to fully exploit the computational power of GPUs. It supports heterogeneous computation where applications off-load parallel portions to the GPU. Typically, CUDA-enabled computation is applied incrementally to existing applications.

Mature scientific applications usually consist of huge source codes and porting such applications to architectures like CUDA includes code refactoring into off-load regions (kernels), dealing with explicit memory management and data- layout optimizations. This makes applications porting timeconsuming, tedious and error-prone. Therefore application porting efforts are often limited in scope to central routines.

Lattice Quantum Chromodynamics (LQCD) is a good example where this porting practice is often applied. LQCD calculations are usually divided into two main parts: The Hybrid Monte Carlo (HMC) gauge field generation part typically requiring capability computing and the analysis part in which the physical observables are determined. For the latter capacity computing will typically suffice. The Chroma application suite [1] for LQCD calculations includes both, the post-Monte Carlo analysis and gauge field generation part.

A common operation to both parts is finding the solution to a large linear system of equations, where the matrix in question is the sparse system that arises from discretizing the Dirac operator. Depending on the simulation parameters, e.g. the quark masses, the linear solves are the operations where most of the compute time is spent and where porting efforts typically focus on. An example of this strategy is the QUDA library [2], which provides CUDA-accelerated linear solvers for common Dirac operator discretizations but whose support beyond this is presently limited.

QUDA has been successfully applied to the post-Monte Carlo analysis phase which is often dominated by the linear solves. However, the gauge field generation part is more diversified and just adding drop-in libraries like QUDA opens a serious Amdahl's law issue. E.g., when deploying the Chroma RHMC $\left(40^{3} \times 256,2+1\right.$ flavors of dynamical quarks, $m_{\pi} \approx 230 \mathrm{MeV}$ ) executing on the $\mathrm{CPU}$ with acceleration only provided through QUDA we measure a speedup factor between $\sim 2.2$ (128 GPUs/CPU sockets) and $\sim 1.8(800$ GPUs/CPU sockets) for our production HMC simulations on Blue Waters. The relatively large fraction of the computation which is not part of a linear solve plus the incurred data transfer between GPU and CPU memories render the gauge field generation part of LQCD calculations not amenable for accelerating solely via the linear solves.

Chroma, however, allows to pursue a different porting strategy. This application is part of a layered software architecture which decomposes its functionality into logical groupings of software components where separate layers or components communicate with each other through clearly defined interfaces. The QCD Data Parallel (QDP++) software layer provides data types and operations suitable for lattice field theory. This is the low-level layer which addresses all characteristics 
of a particular hardware architecture and hides them from the high-level layer. The application layer implements algorithms in terms of the domain-specific data types and operations provided by this interface. Thus, Chroma implements LQCD domain science in a target machine independent way and is as such easier to maintain and extend. It represents a major investment made in software development and its source code consists of over 400,000 C++ code lines.

As a result of the layered structure of the software architecture Chroma can be ported to the CUDA architecture by providing a reimplementation of the low-level layer. This endeavor would have an immediate, large impact as a whole LQCD application suite would instantly tap into the computational power of acceleration as opposed to the traditional incremental application porting. With all computation executing on the accelerator this also would effectively alleviate the effects of Amdahl's law when interfacing to external libraries such as QUDA.

Deploying Chroma with our reimplementation of the lowlevel layer for CUDA we measured a speedup factor of $\sim 11.0$ (128 GPUs/CPU sockets) and $\sim 3.7$ (800 GPUs/CPU sockets) for our production RHMC simulations on Blue Waters.

It is worth noting that the design of the data-parallel level as found in our software architecture is by no means restricted to lattice field theory. This level implements an (embedded) data-parallel language similar to High Performance Fortran (HPF) [3]. We identified the following design elements to be of enabling character for our work: The definition of a data-parallel container, whole-array elemental operations and support for expressions. These are only a few characteristics of data-parallel languages which might be found in other application domains.

In the following we refer to the QDP-JIT/PTX library as the "new implementation" and to the QDP++ library as the "original implementation".

While the expressions which are supported by the interface have a positive impact on science productivity they represent a significant challenge to the library developer when implementing the interface for the CUDA architecture. To date no GPU programming framework is capable to automatically generate accelerated code from expression templates employed in the host program. In order to accelerate expressions advanced annotation or code refactoring, i.e. code separation into CUDA kernel routines, is needed.

However, the structural information about the expressions is encoded in the templates and is accessible at compile time and can be turned into code generators [4]. Thus, we employed a modified version of expression templates in our new implementation so that kernel code generators are built. The code generators, when triggered at runtime, generate CUDA kernels in the PTX language [5]. Those are subsequently translated by the NVIDIA JIT compiler to executable GPU code. This procedure was found to execute smoothly. Neither the code generation, nor the subsequent compilation by the compute compiler generate a significant amount of overhead as we shall demonstrate later on.
Due to CUDA's off-loading execution model user data can be accessed by host and kernel code. Thus the developer is required to explicitly issue memory copy instructions according to the data access pattern of the program. Given the large source code of Chroma, adding memory transfer instructions explicitly would be laborious and error prone to the point of impracticality. To meet the requirements of this dynamic access pattern our new implementation adds comprehensive memory management to the library. Again, we use the expression templates, more precisely the runtime state information stored therein, namely references to data fields. This allows us to make all referenced data fields available in the GPU memory domain prior to launching a kernel. Due to the dynamic nature of these memory accesses we implemented a "cache" for GPU memory which can spill least recently used data fields to CPU memory in order to allocate new fields on the GPU. As a result CUDA memory management is completely automated and altering or annotating the application source code is not needed.

An optimization technique which we applied was changing the data layout for data fields to suit the GPU memory access patterns. We employed a structure of arrays (SoA) data layout such that memory accesses are coalesced. We added a low-overhead auto-tuning step for each compute kernel. This auto-tuning determines experimentally a thread block size, maximizing kernel performance. Another optimization that we applied is overlapping of communication and computation on stencil-like operations.

We found that on the NVIDIA Kepler architecture the generated compute kernels sustain $79 \%$ of the peak memory bandwidth, which is the appropriate metric of efficiency, since the kernels in LQCD computations are memory bandwidth bound.

Our new implementation has allowed us to deploy the full Chroma gauge-generation program in production on large scale GPU-based machines such as Titan at the Oak Ridge Leadership Computing Facility (OLCF), and Blue Waters at the NCSA Petascale Computing Facility. Chroma built on top of the QDP-JIT/PTX library combined with the linear solvers from the QUDA library represent a high-performance configuration for running HMC calculations on GPU-based systems.

This paper is organized as follows. We summarize the necessary background in Sec. III, giving an overview of the scientific domain, discussing the QDP++ application framework, stencil operations and the CUDA architecture. We describe our code generation process in Sec. III giving details on JIT compilation, building code generators and our PTX code generator. Sec. IV and Sec. IV discuss the automatic memory management and the overlapping of communication and kernel computation respectively. We describe the functions discussed in the experimental results and kernel auto-tuning in Sec. VI and Sec. VII We show our numerical results in Sec. VIII and Sec. IX details on related work. We summarize and conclude in Sec. X. 
Table I: Data types in QDP++, REAL $\in\{f l o a t$, double $\}$. The lower part of the table is not part of QDP++'s type system. These data types were added to support the Clover term in the Chroma application.

\begin{tabular}{|c|c|c|}
\hline symbol & definition & type alias \\
\hline$\psi$ & Lattice $<$ Vector $<$ Vector $<$ Complex $<$ REAL $>, 3>, 4>>$ & LatticeFermion \\
\hline$U$ & Lattice $<$ Scalar $<$ Matrix $<$ Complex $<$ REAL $>, 3>>>$ & LatticeColorMatrix \\
\hline$\Gamma$ & Lattice< Matrix< Scalar $<$ Complex $<$ REAL $>>$, 4> > & LatticeSpinMatrix \\
\hline $\begin{array}{l}A_{\text {diag }} \\
A_{\text {tria }}\end{array}$ & $\begin{array}{l}\text { Lattice }<\text { Component }<\text { Diagonal< Scalar }<\text { REAL }>>>> \\
\text { Lattice }<\text { Component }<\text { Triangular }<\text { Complex }<\text { REAL }>>>>\end{array}$ & $\begin{array}{l}\text { n.a. } \\
\text { n.a. }\end{array}$ \\
\hline
\end{tabular}

Table II: Test functions for our benchmarking runs. Notation for gauge, fermion fields as in Table I]

\begin{tabular}{|l|l|l|}
\hline Test & Expression & flop/byte (DP) \\
\hline lcm & $U_{1}=U_{2} * U_{3}$ & 0.458 \\
upsi & $\psi_{1}=U_{1} * \psi_{2}$ & 0.5 \\
spmat & $\Gamma_{1}=\Gamma_{2} * \Gamma_{3}$ & 0.62 \\
matvec & $\psi_{0}=U_{1} * \psi_{1}+U_{1} * \psi_{2}$ & 0.64 \\
\hline clover & $\psi_{0}=A * \psi_{1}$ & 0.525 \\
\hline
\end{tabular}

\section{BACKGROUND}

\section{A. Lattice $Q C D$}

Lattice QCD (LQCD) is the lattice-regularized formulation of Quantum Chromodynamics (QCD), the theory of the strong interaction between quarks and gluons. In LQCD, the continuum theory is typically discretized on a Euclidean spacetime embedded in a hypercubic, 4-dimensional lattice and the QCD path integral over the quark and gluon fields is performed using Monte Carlo methods. LQCD is the most successful, systematic approach to calculate properties of the non-perturbative regime of QCD and is an important tool for nuclear and high-energy physics. A full description of LQCD is beyond the scope of this paper and we refer the reader to the many excellent references available such as [6].

In terms of implementation on a computer we note that there are two primary data "types" in QCD calculations namely the gluon (Gauge) fields which are typically denoted $U_{\mu}(x)$. These variables are complex $\mathrm{SU}(3)$ matrices typically ascribed to the links between lattice sites. Here the coordinate $x$ refers to the lattice site from which the link emanates and $\mu$ is a forward direction $\mu \in\left[0 . . N_{d}-1\right]$ with $N_{d}$ being the number of spacetime dimensions. Quark fields are discretized on lattice sites and can be denoted as $\psi_{C, S}(x)$ where $C$ and $S$ are color and spin indices respectively. Hence, a framework, such as QDP++, needs to provide data types which have indices in the space-time and the internal symmetry (spin, color) domains. Finally we note that nearly all lattice types are represented with complex numbers.

\section{B. The $Q D P++$ Application Framework}

In the following we will introduce concepts of QDP++ which will be necessary in the remainder of the paper. First we will give a very brief overview of the framework and introduce central data types and operations defined in the interface. Finally we will detail some aspects of its implementation.

A central data type is the data-parallel container Lattice which implements the space-time structure of Lattice QCD. This container ascribes its elements logically to grid points on an $N_{d}$-dimensional hypercubic lattice. This container is said to live on the "outer level" of the data type hierarchy and node parallelization, typically with MPI, is implemented on this level. Thus, each node (or rank) maintains a "sub-grid" of the global lattice.

The internal symmetry (spin, color) domains live on the "inner levels" of the hierarchy. The composition of data type levels is implemented with template nesting. Thus a complete data type is composed of four levels, named after the QCD index spaces

\section{Lattice $\otimes \operatorname{Spin} \otimes \operatorname{Color} \otimes$ Complex.}

The interface defines class templates which can be used on inner index spaces as building blocks of the data type system, e.g. Scalar, Vector, Matrix, Complex and Real.

For example, a lattice fermion $\psi$ (we omit the indices here) is a "spin-color vector", i.e. it has vector structure in spin and color space, and is defined as shown in Table I] (upper part). The table shows other commonly used data types. For the user's convenience type aliases are defined for frequently used data types, such as LatticeFermion.

The QDP++ interface defines its operations in operator infix form, i.e. C++ expressions, which can be applied to dataparallel Lattice instances. For example, a multiplication of Scalar $\times$ Vector in spin space, Matrix $\times$ Vector in color space and Complex $\times$ Complex in reality space can be written as:

psi $=u \star p h i ;$

with $u$ of type LatticeColorMatrix and psi,phi of type LatticeFermion. N.B. the operations are implicitly data-parallel, i.e. no loop over the lattice site index has to be written explicitly. The library implements the infix form with expression templates, more precisely with the PETE library [7], [8].

\section{Shift Operations}

In Lattice QCD one is often interested in writing discretized derivative operators such as the Laplacian or Dirac operator. In order to build such operators in a data-parallel language one typically needs stencil-like operations. The QDP++ interface defines so-called shift operations as building blocks for stencil-like computations. These are data-parallel operations that displace the underlying grid points in the specified dimension and direction by one grid point, similar to the circular shift operations in HPF [3].

For example, Figure 1 shows the gauge covariant form of a simple 1-dimensional nearest-neighbor discretization of 
multild<LatticeColorMatrix $>$ u (Nd);

LatticeFermion psi,phi;

psi $=u[m u] *$ shift $(p h i, m u$, FORWARD $)+$

$\operatorname{shift}(\operatorname{adj}(u[m u]) * \operatorname{phi}, \mathrm{mu}, \operatorname{BACKWARD})$;

Figure 1: Simple nearest-neighbor discretization of a partial derivative using the QDP++ interface.

a partial derivative in $\mu$ direction. The vector-like container multild is provided for convenience and (in this example) bundles together the gauge links in different dimensions. Shifting in backward direction $-\mu$ requires multiplication with the Hermitian adjoint of the SU(3) matrix.

Operations including stencil operations provide the opportunity to overlap communication and computation. Communication refers here to inter-MPI rank data transfers. This optimization is implemented in the QDP-JIT/PTX library as described in Sec. V

\section{CUDA Architecture}

The CUDA architecture is a parallel computing platform and programming model created by NVIDIA for use with their GPUs. CUDA C/C++ provides a small set of extensions to standard programming languages, like $\mathrm{C} / \mathrm{C}++$, that enables developers to implement parallel algorithms for GPUs.

The Parallel Thread Execution (PTX) [5] is an intermediate assembler language between high-level CUDA $\mathrm{C} / \mathrm{C}++$ code and GPU machine code. It is a machine-independent virtual machine and instruction set architecture which was made publicly available by NVIDIA. The low-level language interface to kernel programming is a key enabling technology for this work as it has allowed us to implement compute kernels directly in assembly language and interface to a JIT compiler.

Figure 2 shows a schematic of the compilation steps and program representations involved for the GPU kernel part in a typical CUDA C/C++ development chain. Kernel code is extracted from the source code by the NVCC compiler and translated to the PTX language. The NVIDIA compute compile driver (part of the Linux kernel driver) optimizes and translates the PTX kernel program to GPU machine code.

\section{CODE Generation}

The following section describes how the QDP-JIT/PTX library generates GPU kernel code from data-parallel expressions. GPU kernel code is generated using the PTX programming language which we will also refer to as the "secondary language" (translated by the JIT compiler) as opposed to the application or host programming language $(\mathrm{C}++)$.

\section{A. JIT Variables}

First we review the concept of JIT variables. These are quite similar to the familiar variables used in computer programs as they have a type and symbolic names in the secondary language. An important difference, however, is that they are not first-class objects of the host language; they cannot be

\section{CUDA C/C $++\stackrel{\text { NVCC }}{\longrightarrow}$ PTX $\stackrel{\text { Linux driver }}{\longrightarrow}$ GPU code}

Figure 2: Program representations and compilation steps in the NVIDIA CUDA C/C++ development chain. Our compute kernels are implemented directly with the PTX programming language.

stored in an usual variable, neither can they be instantiated at runtime in the execution context of the host program. JIT variables abstract storage locations of the JIT execution context and they are made available to computational manipulation in the host language through reification as manipulable variables in the host language which are often called "JIT values". Manipulating JIT values allows to build computer programs in a secondary language interfacing to a JIT library which provides the arithmetic operations.

\section{B. JIT Data Views}

We introduce "JIT data views" (or simply JIT views) in order to establish a connection between the host program's and the JIT program's execution context. We need to make the abstract $\mathrm{C}++$ container types, i.e. Lattice, visible to the JIT context. In other words, we need to issue the appropriate load and store instructions from and to memory in order to make data values available for arithmetic manipulation in the register file.

Given a sequence container of (structured) elements which are contiguously stored in memory, JIT views provide the means to calculate the index position of a particular data word inside the container. Thus, together with the base memory address of the container's raw data array the index position can be used to calculate the memory offset for the load and store instructions.

The index position is calculated based on a data layout function. This function can be any arithmetic function of the index domains and indices of the domain-specific data types. Since the target architecture is CUDA we use a data layout function that results in coalesced memory accesses:

$$
I\left(i_{V}, i_{S}, i_{C}, i_{R}\right)=\left(\left(i_{R} * I_{C}+i_{C}\right) * I_{S}+i_{S}\right) * I_{V}+i_{V}
$$

where $I_{V}, I_{S}, I_{C}, I_{R}$ are the index domains for, and $i_{V}, i_{S}, i_{C}, i_{R}$ are the indices within these domains for the space-time, spin, color and complex component respectively. The coalescing condition can be easily read off from the above function when assuming thread-parallelisation takes place on the space-time index domain, i.e. adjacent threads (thread number $i_{V}$ ) access adjacent memory locations.

\section{The Code Generation Process}

In the following we describe the building process of code generators as implemented in the QDP-JIT/PTX library. The general concept of generating code via $\mathrm{C}++$ expression templates was pointed out in [4]. Here we will review the basic ideas behind this mechanism as used in our original implementation, and then outline the changes we've employed in our new implementation. 


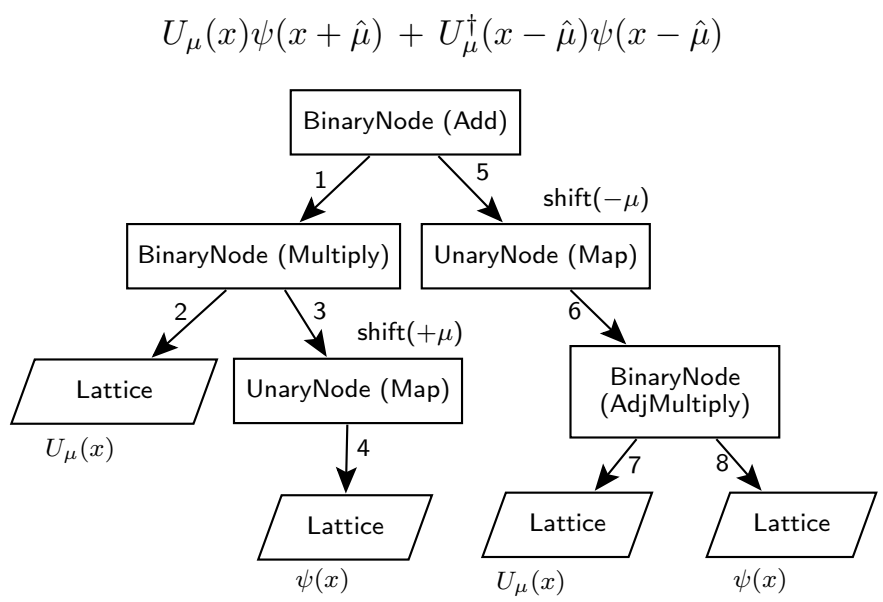

Figure 3: Example of an AST generated by PETE. Inner tree nodes represent operations. Leaf tree nodes refer to data fields. The numbers give the visiting order when "walking the tree".

Expression templates are usually employed in vector libraries with infix operator form in order to efficiently evaluate $\mathrm{C}++$ expressions. Overloaded operators return so-called "proxy objects" which represent parts of the original expression, i.e. unary/binary operations (unary minus, multiplication, etc.). During template instantiation the compiler uses template nesting as the composition operation for proxy objects. The nesting property of proxy objects gives the expression templates a tree structure and the final class template representing the expression can be visualized graphically as a tree, similar to an Abstract Syntax Tree (AST).

Figure 3 shows an example of an AST for the derivative operator shown in Figure 11. Operations (BinaryNode) are represented as inner tree nodes (+,*,Map,AdjMultiply) whereas data fields (Lattice) are represented as tree leaf nodes. The unary map operator implements the shift operations.

In order to evaluate the expression for a particular array index given as a variable $i_{V}$, traditional expression templates traverse the AST in depth-first order, following the numbers as shown in Figure 3, yielding runtime function calls to the operations at the inner nodes (typically inlined by the compiler). Visiting the tree leaf nodes returns the $i_{V}$-th vector element of the container. The loop over the vector index domain is "pulled outside" of the expression, effectively eliminating the vectorsized temporaries typically incurred in naive $\mathrm{C}++$ operator overloading. This unparsing process is also called walking the AST.

In analogy to traditional expression templates the QDP-JIT AST unparser walks the AST, however, instead of yielding runtime function calls to the operations, the unparsing process interfaces to a code generator and yields code that, when executed, generates code in the PTX language for that particular operation. For example, the simple expression $a=b+c$ where $a, b$ and $c$ are vector containers the following (pseudo-) code would be generated

jit_assign( View(a).elem(i_V), jit_add( View(b).elem(i_V), View(c).elem(i_V) ))

Here, jit_assign and jit_add are calls to a code generator (generating PTX in our case) and View: : elem (i_V) creates the earlier introduced JIT views into the vector container pointing at the $i_{V}$-th element. For the CUDA architecture, the loop over the site index is implemented by CUDA thread parallelisation by setting $i_{V}$ to the thread index.

\section{PTX Code Generator}

The last missing piece in the QDP-JIT/PTX code generation chain is the PTX code generator which we will describe below.

Our code generator supports the common arithmetic, bit manipulation and comparison operations on floating-point and integer data types. While $\mathrm{C} / \mathrm{C}++$ supports implicit type conversions, e.g. using mixed precision within a single arithmetic statement is allowed, the PTX language is more restrictive. Legal PTX programs require the proper use of data type conversion instructions. In order to support PTX code generation from mixed precision expressions we added implicit type promotion. Thus data type conversion instructions are silently issued as needed.

Employing the PTX language for writing programs in scientific computing has one major downside: The mathematical functions, i.e. those from the $\mathrm{C}$ math library, are not available. Only a small subset of these functions, the so-called "fastmath" functions, are supported. These are functions for which fast, but reduced accuracy approximations are implemented directly on the GPU hardware.

As a work-around, we generated PTX kernel programs for the relevant mathematical functions with CUDA's static compilation tools (NVCC). Based on this set of kernels we manually created PTX subroutines for each of the functions. The code generator will silently issue calls to the appropriate subroutine every time a mathematical function is requested. The downside of providing pre-generated PTX implementations in such a way is that their implementation is tied to a particular version of the PTX language and as a result a recompilation is needed every time the PTX standard changes.

An advantage of implementing GPU kernels in the PTX language is that the NVIDIA JIT compiler translates the kernels very quickly. JIT compilation overhead for our compute kernels was found to be between 0.05 and 0.22 seconds on the $12 \mathrm{k}$ compute node as specified in the experimental result section, Sec. VIII.

\section{Automated Memory MANAGEMENT}

The CUDA architecture implements an execution model where the GPU portion of the program code is off-loaded as kernels to the accelerator while non-kernel code executes on the CPU. Thus our data structures such as Lattice are accessible by kernel and host code and it's part of the library developers' responsibilities to issue the appropriate memory transfer and synchronization instructions well before the actual access. 
The QDP-JIT/PTX library automates this process by using a software implementation of a cache. Prior to a kernel launch for a given expression, we walk its AST, e.g. the one shown in Figure. 3, extract the references to data fields stored at the leaf tree nodes and "cache" (make available in GPU memory) the according data fields. All data fields referenced by that particular kernel are then available in GPU memory.

Data fields are "paged-out" (copied to CPU memory) either when they are accessed by CPU code or upon a caching event that cannot be serviced immediately due to too many cached data fields. The problem of when to page-out a particular data field is decided by a "least recently used" spilling algorithm based on the timestamp of the last reference from a compute kernel.

\section{OVERLAPPING MPI COMMUNICATION AND COMPUTATION}

On distributed memory systems the shift operations introduce data dependencies on off-node grid points. This provides the opportunity to overlap off-node communication with kernel computation. Since the node-local sub-grid is logically shaped as a $N_{d}$-dimensional hypercubic grid the face sites, i.e. the sites to send off-node, can be easily determined for a shift operation in a particular dimension and direction.

For a given expression which includes shift operations the local sub-grid is partitioned into "inner sites" and "face sites". Compute kernels gather data into a contiguous region of GPU memory from where it's sent directly (MPI) to the destination node. For MPI implementations that are not "CUDA-aware" the data is first copied to CPU memory. While off-node data is in transition the compute kernel is launched on the inner sites and after data has been received the remaining sites are evaluated. We do not detail much further on the implementation as this technique is well established, for example see [9], [10].

Our implementation is limited to simple shift operations as used, e.g., in first-order finite difference operators. Operations involving next-to-nearest neighboring site communications, i.e. "shifts of shifts", execute the inner-most shift operations in a non-overlapping fashion.

\section{TEST FunCtions}

Table II lists the test functions that we selected for our benchmark tests. These functions are relevant to LQCD calculations as they are used in, e.g., calculations with SU(3) gauge fields, fermionic force terms and spin projections. The functions were chosen to exercise different combinations of index spaces.

\section{A. Custom User-Defined Functions}

In LQCD, the so-called clover term [11] can be added to the Wilson Fermion action in order to achieve $O\left(a^{2}\right)$ discretization errors. The clover term is a local operator defined as

$$
A(x)=1 / 4 c_{\mathrm{SW}} \sigma_{\mu \nu} F_{\mu \nu}(x)
$$

where the field strength tensor $F_{\mu \nu}(x)$ acts in color space and $\sigma_{\mu \nu}$ in spin space.
In our particular spin basis (of $\gamma_{\mu}$ matrices) the clover term is Hermitian, and splits into a block diagonal form. There are two blocks, corresponding to the first two and the second two spin components respectively of the overall four components available. For each spin components we also have 3 color components giving each block dimension 6 overall. We store each block as the 6 real numbers along the diagonal, and the 15 complex numbers making up the lower triangular sub-diagonal part of the block. The upper (super-diagonal) part can be found by Hermitian conjugation (transpose and complex conjugation) as needed.

However, this implementation of the clover term mixes spin and color index spaces in a non-trivial way and hence typical implementations address this term outside of the QDP++ library since there calculations in different index spaces are strictly separated from each other. Our code generation process, however, supports user-defined operations even if they mix the spin-color structure. We added support for the clover term on the application level, i.e. in Chroma. In terms of the nested QDP++ template structure, we use the template level reserved for the color indices to store the triangular part of a single block, and employ the template level usually used for spin indices for the index of the two separate blocks. See Table I (lower part) for how our implementation makes use of the spin and color spaces for the clover term.

\section{KERNEL AUTO-TUNING}

In general, the performance of GPU kernels is impacted by factors such as how the computation is organized into threads, and groups of threads; in particular on the number of threads in a group, known as the "thread block size". However, the compute kernels generated by our code generator are simple streaming kernels, which have no thread block communication and their performance should have only a weak dependence on the thread block size as long as enough threads are in flight. Empirically we found, as expected, that on the Kepler architecture running with a thread block size of at least 128 our streaming kernels achieve the highest performance.

Running all the generated kernels with the same fixed thread block size will likely not result in the most optimal performance, since different kernels will have different resource requirements (e.g. in terms of registers). Thus the same block size may result, for example, in different levels of GPU utilization for different kernels. Indeed some kernels may even exhaust resources and fail to launch altogether. Therefore we tune the thread block size automatically on a per kernel basis.

Our auto-tuning strategy is implemented as follows: First we try to launch a given kernel with the maximum thread block size allowed for the GPU in question (we use 1-dimensional blocks, thus $2^{10}$ for Kepler) and, if that fails, re-try, having reduced the thread block size by a factor of 2 until the launch succeeds. Once successfully launched, consecutive launches "probe" smaller block sizes until the execution time increases significantly (arbitrarily we use 33\%). The "best configuration" would then be used for all consecutive launches. We found that this auto-tuning method comes with very little overhead: No 


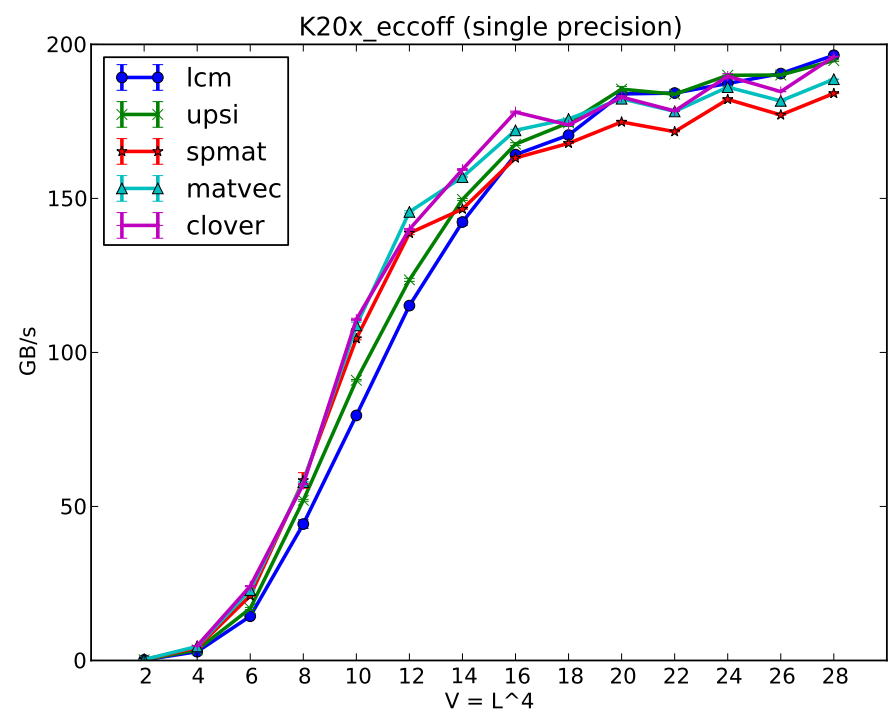

Figure 4: Comparison performance of benchmarking kernels in single precision on Tesla K20x (ECC disabled).

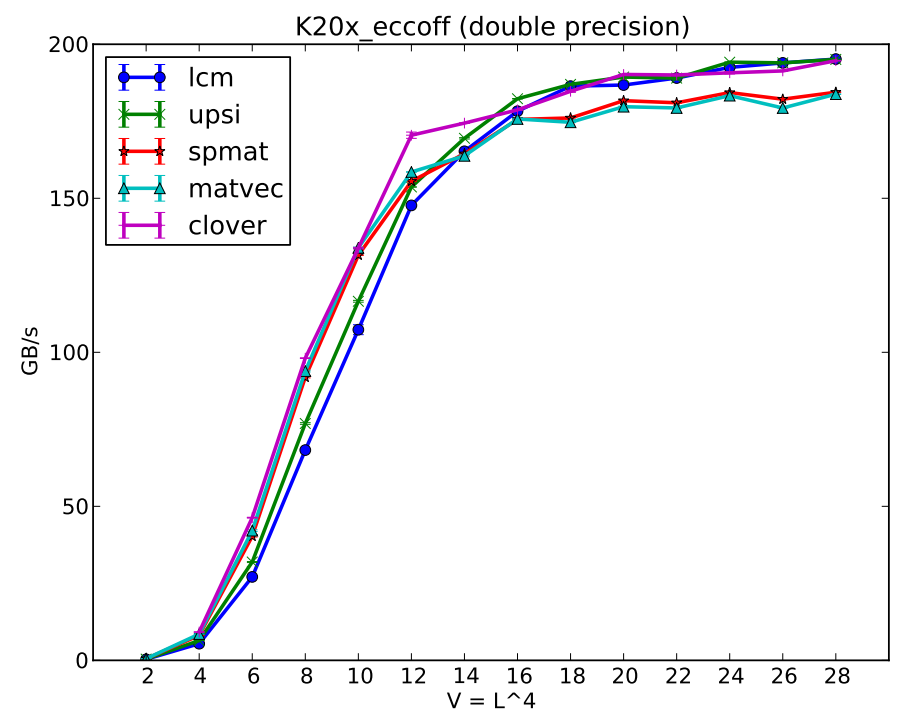

Figure 5: Comparison performance of benchmarking kernels in double precision on Tesla K20x (ECC disabled).

kernels are launched solely for the purpose of tuning; kernel tuning is carried out on the payload compute launches.

\section{EXPERIMENTAL RESULTS}

\section{A. Hardware description}

Our experimental results were acquired on the Jefferson Lab $12 \mathrm{k}$ cluster. Nodes of this cluster contain dual-socket Xeon E5-2650 CPUs running at $2.0 \mathrm{GHz}$. The nodes run CentOS 6.2. We used a node with four NVIDIA K20x GPUs (GK110 architecture) installed. The K20x has as a peak performance of 1.3 TFlops (DP) and a peak memory bandwidth of 250 $\mathrm{GB} / \mathrm{sec}$ (ECC disabled). We used the CUDA toolkit version 5.5 and the NVIDIA UNIX x86-64 Kernel Module version 319.37. For the 2 GPUs benchmark run detailed in Subsec.

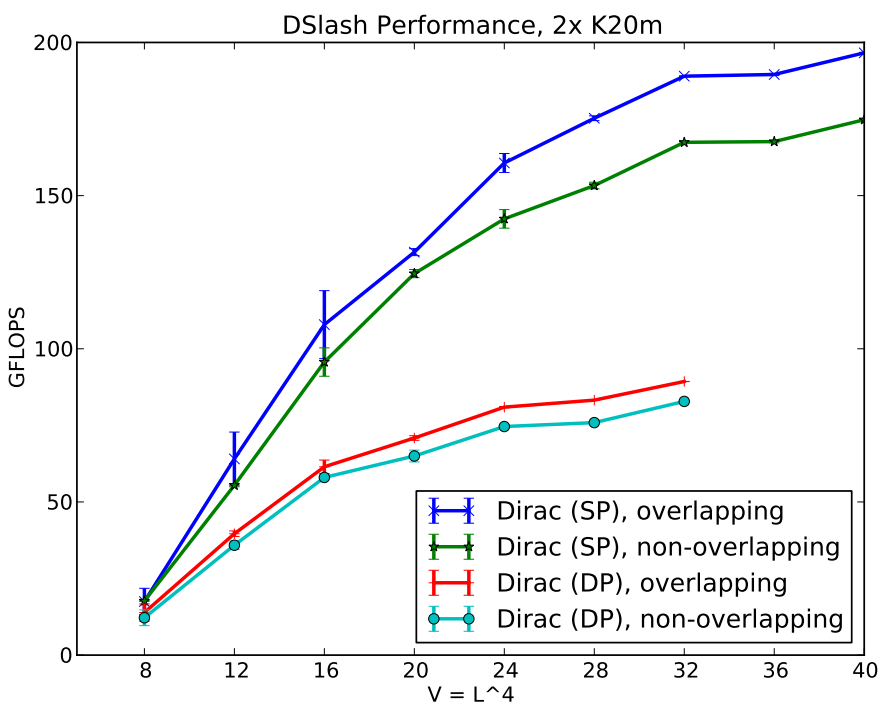

Figure 6: Performance of the hopping part of the Wilson Dirac operator. Comparison when overlapping of inter-GPU communication and computation is enabled and disabled. Using 1 NVIDIA K20m per node (ECC enabled).

VIII-C we used $2 \mathrm{~K} 20 \mathrm{~m}$ nodes with MVAPICH2 version 1.9 with CUDA-aware MPI.

Our application benchmark on multiple GPUs was run on the XK cabinets of the NCSA BlueWaters system and on the Titan Supercomputer at the OLCF. These nodes comprise 1 AMD 6276 Interlagos Processors (8 physical cores) and 1 NVIDIA GK110 Kepler accelerator. The CPU comparison run was deployed on XE nodes which comprise 2 AMD 6276 Interlagos Processors per node.

\section{B. Single GPU Performance}

We note that our test functions (Sec. VI) on the K20x architecture are memory bandwidth bound in single and double precision (SP, DP). Thus, we are interested in the fraction of the memory bandwidth that our kernels sustain on that hardware vs. the theoretical maximum memory bandwidth. However, since the sustained bandwidth and performance are directly related we will use both synonymously.

Figure 4 and 5 show the sustained bandwidth as a function of the local sub-grid size and for SP and DP respectively. The curves corresponding to different kernels show a universal behavior and (nearly) fall on top of each other indicating that the performance of our generated code depends very little on the actual function which it implements.

In SP and DP for smaller volumes the sustained bandwidth is steadily increasing with the volume up to a "shoulder region" at around $V=16^{4}$ for SP and $V=12^{4}$ for DP from where on the performance increases only little. For the largest volumes the kernels sustain about $79 \%$ of the theoretical maximum memory bandwidth.

The shape of the performance curve is what one would expect. The GPUs requires many threads resident to a single Streaming Multiprocessor (SM) in order to effectively hide latency of memory accesses. The shoulder region seems to 
indicate a "thread saturation" of SMs. Below the shoulder region not enough thread blocks are resident and consequently the sustained memory bandwidth decreases.

\section{Overlapping Inter-GPU Communication}

In the following we demonstrate the impact of overlapping communication and computation as detailed in Sec. V

The hopping part of the Wilson discretization of the Dirac operator

$$
H\left(x, x^{\prime}\right)=\sum_{\mu=1}^{4}\left(1-\gamma_{\mu}\right) U_{x}^{\mu} \delta_{x+\hat{\mu}, x^{\prime}}+\left(1+\gamma_{\mu}\right) U_{x-\hat{\mu}}^{\mu \dagger} \delta_{x-\hat{\mu}, x^{\prime}}
$$

was implemented using the high-level domain abstractions provided by the QDP-JIT/PTX library.

For this test we used a total of two NVIDIA K20m installed in two $12 \mathrm{k}$ compute nodes, i.e., we see overlapping of compute kernels with MPI communication routed through the PCIe bus and the InfiniBand interconnect.

Figure 6 shows the combined performance of the hopping part as a function of the global volume. Shown is the comparison of the performance when overlapping is enabled or disabled, in SP and DP. For SP we measured an $11 \%$ performance increase for the largest volume. The impact on DP performance is smaller, about $7 \%$ for the largest volume. This indicates that overlapping of MPI communication and CUDA kernel computation takes place.

We would like to note that this benchmark test was included solely to demonstrate the effect of overlapping communication and computation. It is not meant to compete with implementations which focus optimizations exclusively to this operator. Our implementation of the hopping term was generated from its high-level representation.

We measured the same operator as available from the QUDA library (version 0.6) [12] on the same hardware. We configured QUDA with "overlapping communications", targeting compute capability " 3.5 ". In SP, $V=40^{4}$ we measured 346 GFLOPS (with uncompressed gauge fields) where our implementation achieves 197 GFLOPS (speedup factor 1.76). In DP, $V=32^{4}$ we measured 171 GFLOPS where our implementation achieves 90 GFLOPS (speedup factor 1.9). QUDA can apply other optimizations to the Dslash operator like reconstruction of $\mathrm{SU}(3)$ matrices from 8 or 12 real numbers to gain additional speedups. However, we used a set of optimizations such that the same amount of work was done by QUDA and our implementation since we are focusing here on communications. These results give an indication of the amount of "headroom" available for hand tuned optimizations, without employing gauge compression.

\section{Hybrid Monte Carlo on Blue Waters}

Our implementation enables us to deploy the full Chroma gauge-generation program on large scale GPU-based machines. Using additionally the linear solvers from the QUDA library [2] results in a high-performance configuration for running HMC calculations on systems such as Titan at the OLCF, and Blue Waters at the NCSA.

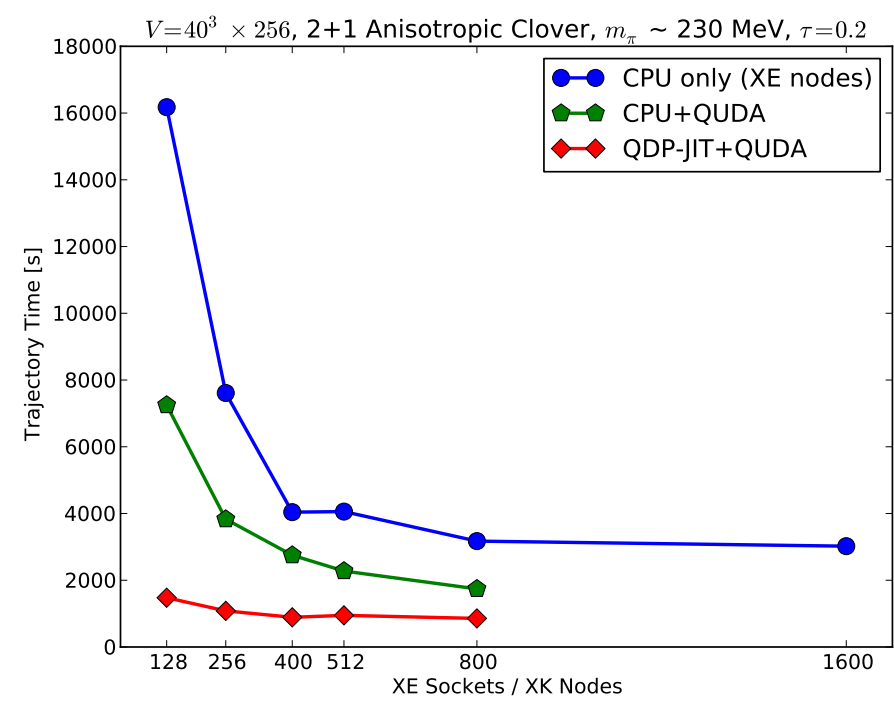

Figure 7: Strong scaling of HMC on Blue Waters.

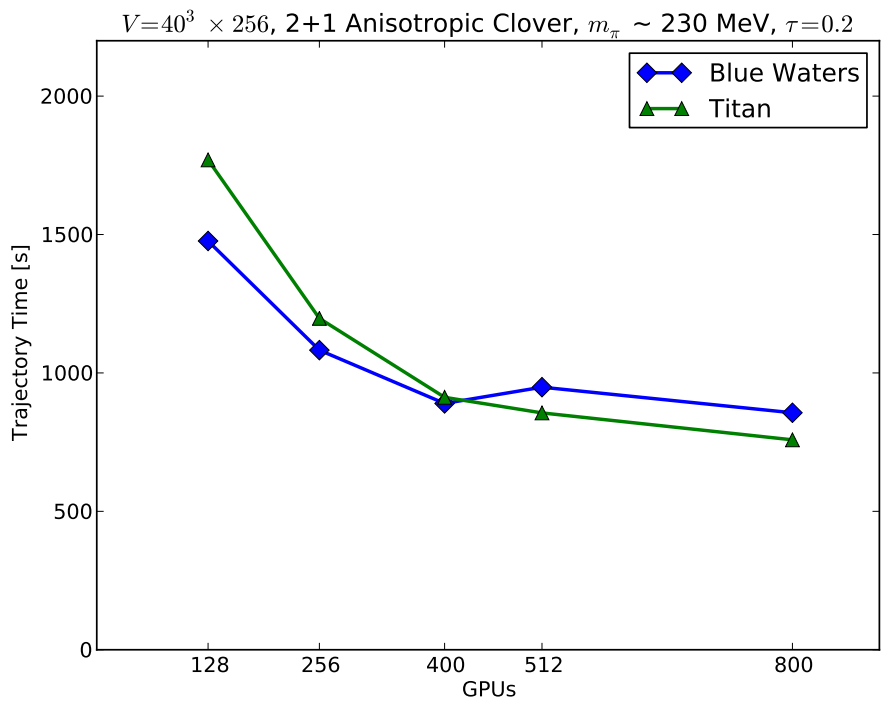

Figure 8: Comparison Blue Waters and Titan. Shown is the strong scaling of HMC for the configuration "QDP-JIT+QUDA".

We are using QUDA's device interface to call-out from Chroma to the linear solvers. The interface supports the optimized data layout as used in the QDP-JIT/PTX library and thus eliminates the requirement to copy the spinor, gauge and clover fields to the CPU memory and changing the data layout prior to calling the solvers.

We will now discuss our scaling results which we obtained using exactly the same simulation parameters as we are using in our production program, modulo shortening the trajectory lengths. The global lattice size is set to $V=40^{3} \times 256$. We are running simulations with $N_{f}=2+1$ dynamical flavors of Wilson clover [11] fermions on an anisotropic lattice. We employ mass preconditioning [13] and the rational approximation [14] to calculate the determinant of the Dirac operator with the strange quark mass.

Chroma was deployed throughout in double precision on 
Blue Waters in several software configurations. The individual configurations differ by how the GPUs are utilized for different parts of the calculation. Figure 7 shows the time for a trajectory for the various software configurations.

In order to get a baseline of performance we deployed Chroma in a CPU only configuration. We varied the number of XE sockets from 128 to 1600 . We found good scaling up to $400 \mathrm{XE}$ sockets from where on performance shows only modest increase; going from 800 to 1600 has only a marginal effect.

Plugging in the QUDA linear solvers, i.e. configuration "CPU+QUDA", has a positive impact on the performance; the speedup factor over the whole range of partition sizes stays roughly constant; we measured a speedup factor of $\sim 2.2$ for $128 \mathrm{GPUs} / \mathrm{CPU}$ sockets and a factor of $\sim 1.8$ for 800 GPUs/CPU sockets. However the overall scaling trend has not improved. This configuration is sensitive to Amdahl's law effects combined with the poorer scaling of the GPU part due to repeated copying of data fields between the CPU and the GPU and changing data layouts.

Turning to a Chroma build over QDP-JIT/PTX resulted in generating about $200 \mathrm{GPU}$ kernels for this particular trajectory. Based on our earlier measurements we estimate the total JIT compilation overhead to be around $\sim 10-30$ seconds, and thus negligible compared to the time needed for the trajectory.

The configuration "QDP-JIT+QUDA" achieves the highest performance of all deployed configurations. Full benefit is taken from the algorithmic improvements (QUDA GCR solver) due to the seamless interface between the QUDA and QDP-JIT/PTX libraries, and the fact that all computation is accelerated effectively alleviates the effects of Amdahl's law. A speedup factor of $\sim 11.0$ could be achieved on 128 GPUs/CPU sockets, and a significant factor of $\sim 3.7$ on 800 GPUs/CPU sockets. When compared to the "CPU+QUDA" configuration, "QDP-JIT+QUDA" achieves a speedup factor of $\sim 2.0$ for 800 GPUs.

Figure 8 shows the timing for the trajectory on Blue Waters and the Titan supercomputer. As expected the Blue Waters and Titan results are hardly distinguishable when bearing in mind that our benchmark timings on these systems typically show some amount of fluctuation.

The most efficient machine size for the $40^{3} \times 256$ lattice size is $128 \mathrm{XK}$ nodes where the integrated resource costs are 258 vs. 52 node hours for the "CPU + QUDA" and "QDPJIT + QUDA" configurations respectively. Thus, due to this work the computational cost for our HMC simulations with this lattice size could be reduced by a factor of $\sim 5$.

\section{Related Work}

Automatic GPU code generation was addressed, e.g., in [15], [16]. There, however, kernel code is generated using CUDA C++. This creates a dependency on calling NVCC at runtime. Previously we used a similar approach [17], [18] and our findings were that this does not lead to a smooth process. The NVCC compiler, designed for static compilation, is not fast in translating code. Thus caching of compiled kernels on the filesystem is desired. Also calling NVCC on the compute node, is not possible in all environments, e.g. on Cray compute node Linux environment. Previous efforts completely ignore GPU memory management, optimizations such as optimizing the data layout, kernel auto-tuning and overlapping of computation and communication.

Development of an LQCD application using OpenCL was reported in [19]. All operations involved in an HMC simulation were implemented separately as kernels. This work supports single GPUs only and reports sustaining between $77 \%$ and $80 \%$ of the peak memory bandwidth.

Porting (parts of) LQCD calculations to the CUDA architecture was addressed in previous work [9], [10], [2], [20], [21], [22], [23]. However, these efforts belong to the class of traditional porting as focus was limited to only specific parts of LQCD calculations.

\section{COnClusions}

The layered structure of the Chroma software architecture allowed us to provide a reimplementation of the low-level layer and with it to port the whole application layer to the CUDA architecture. The reason why this was tractable is that this low-level layer implements a data-parallel language with the use of expression templates. Those make the structure of operations accessible to compile-time computations which allowed us to build kernel code generators and automate the CUDA memory management. A necessity was the availability of a JIT compiler for the CUDA architecture.

Due to this work we reduced the computational cost for our production Hybrid Monte Carlo simulations on Blue Waters and Titan by a factor of $\sim 5$. While this worked very well for the scientific domain of lattice QCD, especially for Chroma, we expect the approach can also benefit in other domains with data-parallel computational patterns.

\section{Future WORK}

We are exploring the possibility to interface to a compiler framework such as LLVM [24]. This would allow us to target other architectures as well.

\section{ACKNOWLEDGMENTS}

We gratefully acknowledge support for this work on OLCF Titan through Directors Discretionary Allocation LGT006 (2012-2013) and through the INCITE project Allocation LGT003 2012-2013.

This research is part of the Blue Waters sustained-petascale computing project, which is supported by the National Science Foundation (award number OCI 07-25070) and the state of Illinois. Blue Waters is a joint effort of the University of Illinois at Urbana-Champaign and its National Center for Supercomputing Applications.

Partial support for this work was provided through the Scientific Discovery through Advanced Computing (SciDAC) program funded by U.S. Department of Energy, Office of Science, Offices of Advanced Scientific Computing Research, Nuclear Physics and High Energy Physics. 
This research was in part supported by the Research Executive Agency (REA) of the European Union under Grant Agreement number PITN-GA-2009-238353 (ITN STRONGnet).

Notice: Authored by Jefferson Science Associates, LLC under U.S. DOE Contract No. DE-AC05-06OR23177. The U.S. Government retains a non-exclusive, paid-up, irrevocable, world-wide license to publish or reproduce this manuscript for U.S. Government purposes.

\section{Code Availability}

The latest version of the QDP-JIT/PTX library is always available in a publicly-accessible source code repository [25].

\section{REFERENCES}

[1] SciDAC Collaboration, LHPC Collaboration, UKQCD

Collaboration Collaboration, R. G. Edwards and B. Joó, "The Chroma software system for lattice QCD," Nucl.Phys.Proc.Suppl. 140 (2005) 832 arXiv: hep-lat/0409003 [hep-lat]

[2] M. A. Clark, R. Babich, K. Barros, R. C. Brower, and C. Rebbi, "Solving Lattice QCD systems of equations using mixed precision solvers on GPUs ," Comput. Phys. Commun. 181 (2010) 1517-1528 arXiv:0911.3191 [hep-lat]

[3] D. B. Loveman, "High Performance Fortran," IEEE Parallel Distrib. Technol. 1 no. 1, (Feb., 1993) 25-42 http://dx.doi.org/10.1109/88.219857

[4] T. L. Veldhuizen, "C++ Templates as Partial Evaluation," CoRR cs.PL/9810010 (1998) .

[5] NVIDIA, "Parallel Thread Execution ISA Version 3.1," http://docs.nvidia.com/cuda/pdf/ptx_isa_3.1.pdf

[6] M. Creutz, Quarks, gluons and lattices. Cambridge University Press, Cambridge, New York, Melbourne, 1983.

[7] T. Veldhuizen, "Expression Templates ," C++ Report 7, 1995

[8] S. Haney, J. Crotinger, S. Karmesin, and S. Smith, "Easy expression templates using PETE, the Portable Expression Template Engine," Technical Report LA-UR-99 (1999) 777.

[9] R. Babich, M. A. Clark, B. Joó, G. Shi, R. C. Brower, and S. Gottlieb, "Scaling Lattice QCD Beyond 100 GPUs," in Proceedings of 2011 International Conference for High Performance Computing, Networking, Storage and Analysis, SC '11, pp. 70:1-70:11. ACM, New York, NY, USA, 2011. http://doi.acm.org/10.1145/2063384.2063478

[10] R. Babich, M. A. Clark, and B. Joó, "Parallelizing the QUDA Library for Multi-GPU Calculations in Lattice Quantum Chromodynamics," in Proceedings of the 2010 ACM/IEEE International Conference for High Performance Computing, Networking, Storage and Analysis, SC '10, pp. 1-11. IEEE Computer Society, Washington, DC, USA, 2010. arXiv:1011.0024 [hep-lat] http://dx.doi.org/10.1109/SC.2010.40

[11] S. B. and W. R., "Improved continuum limit lattice action for QCD with Wilson fermions," Nucl. Phys. B 259 (1985) 572.

[12] https://github.com/lattice/quda

[13] M. Hasenbusch and K. Jansen, "Speeding up lattice QCD simulations with clover improved Wilson fermions," Nucl.Phys. B659 (2003) 299-320, arXiv:hep-lat/0211042 [hep-lat]

[14] M. Clark, A. Kennedy, and Z. Sroczynski, "Exact 2+1 flavour RHMC simulations," Nucl.Phys.Proc.Suppl. 140 (2005) 835-837 arXiv: hep-lat/0409133 [hep-lat]

[15] J. Chen, B. Joo, W. Watson III, and R. Edwards, "Automatic Offloading C++ Expression Templates to CUDA Enabled GPUs," in Proceedings of the 2012 IEEE 26th International Parallel and Distributed Processing Symposium Workshops \& PhD Forum, IPDPSW '12, pp. 2359-2368. IEEE Computer Society, Washington, DC, USA, 2012. http://dx.doi.org/10.1109/IPDPSW.2012.293

[16] J. M. Cohen, "Processing Device Arrays with C++ Metaprogramming," in GPU Computing Gems Jade Edition, W. Hwu, ed., Applications of GPU Computing Series. Elsevier Science, 2011

[17] F. Winter, "Gauge Field Generation on Large-Scale GPU-Enabled Systems," PoS LATTICE2012 (2012) 185, arXiv:1212.0785 [hep-lat]

[18] F. Winter, "Accelerating QDP++/Chroma on GPUs," PoS LATTICE2011 (2011) 50, arXiv:1111.5596 [hep-lat]
[19] M. Bach, V. Lindenstruth, O. Philipsen, and C. Pinke, "Lattice \{QCD based on opencl," Computer Physics Communications 184 no. 9, (2013) 2042 - 2052 http://www.sciencedirect.com/science/article/pii/S0010465513001288

[20] A. Alexandru, M. Lujan, C. Pelissier, B. Gamari, and F. Lee, "Efficient Implementation of the Overlap Operator on Multi-GPUs,"] in Proceedings of the 2011 Symposium on Application Accelerators in High-Performance Computing, SAAHPC '11, pp. 123-130. IEEE Computer Society, Washington, DC, USA, 2011. http://dx.doi.org/10.1109/SAAHPC.2011.13

[21] G. I. Egri, Z. Fodor, C. Hoelbling, S. D. Katz, D. Nogradi, et al., "Lattice QCD as a Video Game," Comput.Phys.Commun. 177 (2007) 631-639 arXiv:hep-lat/0611022 [hep-lat]

[22] C. Bonati, G. Cossu, M. D'Elia, and P. Incardona, "QCD Simulations with Staggered Fermions on GPUs," Comput.Phys.Commun. 183 (2012) 853-863 arXiv:1106.5673 [hep-lat]

[23] M. Wagner, "GPUs Immediately Relating Lattice QCD to Collider Experiments." http://on-demand.gputechconf.com/gtc/2013/ presentations/S3153-Lattice-QCD-Collider-Experiments.pdf 2013. GTC 2013.

[24] C. Lattner and V. Adve, "LLVM: A Compilation Framework for Lifelong Program Analysis \& Transformation," in Proceedings of the international symposium on Code generation and optimization: feedback-directed and runtime optimization, CGO '04, pp. 75-. IEEE Computer Society, Washington, DC, USA, 2004. http://dl.acm.org/citation.cfm?id=977395.977673

[25] https://github.com/fwinter/qdp-jit 